\title{
Acute Intrathoracic Gastric Volvulus due to Diaphragmatic Hernia: A Rare Emergency Easily Overlooked
}

\author{
Hyung Hun Kim Seun Ja Park Moo In Park Won Moon \\ Department of Internal Medicine, Kosin University College of Medicine, Busan, \\ Korea
}

\section{Key Words}

Gastric volvulus · Diaphragmatic hernia · Torsion · Emergency

\begin{abstract}
Acute intrathoracic gastric volvulus occurs when the stomach undergoes organoaxial torsion in the chest due to either concomitant enlargement of the hiatus or a diaphragmatic hernia. latrogenic diaphragmatic hernia can occur after hiatal hernia repair and other surgical procedures, such as nephrectomy, esophagogastrectomy and splenopancreatectomy. We describe a 49-year-old woman who presented to our emergency department with acute moderate epigastric soreness and vomiting. She had undergone extensive gynecologic surgery including splenectomy 1 year before. The chest radiograph obtained in the emergency department demonstrated an elevated gastric air-fluid level in the left lower lung field. An urgent gastroscopy showed twisted structural abnormality of the stomach body. A computed tomography scan demonstrated the distended stomach, located in the left lower hemithorax through a left diaphragmatic defect. Emergent transthoracic repair was performed. Postoperative recovery was uneventful, and the patient did not experience any pain or difficulty with eating.
\end{abstract}

\section{Introduction}

Gastric volvulus is defined as rotation of the stomach or part of the stomach by more than $180^{\circ}$ creating a closed-loop obstruction [1]. Mostly, it happens in the fifth decade of life. It can be classified by anatomy, etiology or the axis of rotation. The most common type in adults is the organoaxial type, which means that the stomach rotates along the longitudinal axis. Acute intrathoracic gastric volvulus occurs when the stomach undergoes organoaxial torsion in the chest due to either concomitant enlargement of the 
hiatus or a diaphragmatic hernia [2]. An iatrogenic diaphragmatic hernia can occur following surgical procedures, such as left nephrectomy [3], esophagogastrectomy [4] and splenopancreatectomy [5], although it most frequently develops after hiatal hernia repair by either the Nissen or the Allison techniques. The common symptoms of intrathoracic stomach are postprandial chest discomfort, dysphagia, vomiting, hemorrhage, chest fullness, inability to belch, and anemia; reflux alone is uncommon, and the signs and symptoms of acute gastric volvulus include abdominal pain and distention, especially in the upper abdomen, and vomiting with progression to nonproductive retching. It is traditionally diagnosed by seeing intrathoracic viscera in the chest radiograph; this can be followed by a barium contrast study or upper gastrointestinal endoscopy. Currently, computed tomography (CT) scan can lead to an immediate diagnosis with all the anatomical details. This disease is potentially life-threatening as delayed diagnosis and treatment may result in perforation, infarction or other lethal insults [6]. Due to its rarity, however, an individual physician who has no personal experience with this disease can potentially misdiagnose it as a nonsurgical gastrointestinal disease. Here, we describe a rare complication of gynecologic surgery, an acute intrathoracic gastric volvulus due to diaphragmatic hernia occurring in a middle-aged woman who underwent total adnexal hysterectomy, salpingo-oophorectomy, omentectomy and splenectomy for treatment of cervical cancer one year earlier.

\section{Case Report}

A 49-year-old woman presented to our emergency department with a one-day history of acute moderate epigastric soreness and vomiting. She had undergone total adnexal hysterectomy and salpingo-oophorectomy secondary to cervical cancer one year previously; omentectomy and splenectomy had also been performed. Following these operations, she had completed 9-cycle chemotherapy and had visited our hospital regularly. Upon arrival in the emergency department, physical examination revealed diffuse tenderness over the epigastric area. Bowel sounds were hyperactive. The initial electrocardiograph was unremarkable. Her blood pressure was $110 / 85 \mathrm{~mm} \mathrm{Hg}$, heart rate was 130 beats per minute, respiratory rate was 15 times per minute, and body temperature was $36.8^{\circ} \mathrm{C}$. Initial laboratory results were as follows: white blood cell count $7,600 / \mu \mathrm{l}$ with $41 \%$ segmented neutrophils, blood urea nitrogen $20 \mathrm{mg} / \mathrm{dl}$, creatinine $0.5 \mathrm{mg} / \mathrm{dl}$, amylase $38 \mathrm{IU} / \mathrm{l}$, lipase $10 \mathrm{IU} / \mathrm{l}$ and C-reactive protein $0.53 \mathrm{mg} / \mathrm{l}$. In contrast to a chest radiograph made three months earlier, the chest radiograph obtained in the emergency department demonstrated an elevated gastric air-fluid level in the left lower lung field (fig. 1). At first, this finding was regarded as an eventration of the diaphragm with the stomach present under the diaphragm. As the patient complained of vomiting and epigastric soreness, an urgent gastroduodenoscopy was performed. Gastroscopy demonstrated massive fluid collection just below the gastroesophageal junction and pseudo-obstruction due to a structural abnormality of the stomach body (fig. 2). The endoscope was not able to reach the duodenum due to this structural abnormality. These findings suggested an acute gastric volvulus. A chest CT scan demonstrated the distended stomach, located in the left lower hemithorax through a left diaphragmatic defect (fig. 3). With a working diagnosis of acute gastric volvulus with severe obstruction, emergent transthoracic repair was performed. The stomach was found in the left lower thorax with adhesions among the diaphragm, herniated stomach and left lower lobe of the lung. The herniated stomach was reduced into the abdomen, and the diaphragm was repaired following lysing of adhesions. Postoperative recovery was uneventful, and the patient did not experience any pain or difficulty with eating.

\section{Discussion}

Acute gastric volvulus is a rare disease that requires a high index of suspicion for diagnosis and rapid treatment [2]. The signs and symptoms of gastric volvulus depend on 
the rapidity of onset and the degree of rotation and obstruction. This disease is potentially life-threatening because delayed diagnosis and treatment may lead to perforation, infarction or other lethal insults [7]. Because of the rarity of this disease, common gastrointestinal complaints may lead the physician to misdiagnose this as a nonsurgical gastrointestinal disease if detailed history and physical examination are not obtained [8].

The classic triad described by Borchardt [9] of retching, severe and constant epigastric pain, and difficulty in inserting a nasogastric tube suggests an acute gastric volvulus. Traditionally, it is diagnosed by observation of intrathoracic viscera on chest radiograph, followed by a barium contrast study or upper gastrointestinal endoscopy [8]. When our patient presented to the emergency unit, a chest radiograph may have demonstrated diaphragmatic hernia, but this was interpreted as the stomach being elevated with eventration of the diaphragm, leading to the misdiagnosis of the stomach being present under the diaphragm. We entertained the possibility of a gastric volvulus after gastroscopy and demonstrated a twisted stomach. A chest CT scan was then obtained to evaluate for intrathoracic gastric volvulus with diaphragmatic hernia. A CT scan can lead to an immediate diagnosis, given its level of anatomical detail [10]. Upon $\mathrm{CT}$, the stomach in an unusually high position or with an abnormal axis in a patient with acute abdominal pain and vomiting should make one suspect gastric volvulus [11]. In our case, the fundus was in the thoracic cavity, and the body of the stomach was extremely twisted. When the fundus was rotated back into the abdominal cavity, it caused a closed loop and acute volvulus of the stomach. Surgery is mandatory whenever a diagnosis of diaphragmatic injury or acute volvulus is made $[5,12]$.

The pathogenesis of this case was atypical and iatrogenic. Iatrogenic diaphragmatic hernia can occur following surgical procedures, such as left nephrectomy [3], esophagogastrectomy [4] and splenopancreatectomy [5], although it most frequently develops after hiatal hernia repair by either the Nissen or the Allison techniques. The patient had undergone extensive abdominal operations: total adnexal hysterectomy, salpingo-oophorectomy, omentectomy and splenectomy for treatment of cervical cancer one year earlier. We assume that at that time a small and intraoperatively unrecognized injury of the diaphragm occurred. During the ensuing period, this laceration enlarged progressively, likely due to the observed adhesions, with resulting traction on the diaphragm [13-16]. Following this, the stomach migrated into the chest through the resulting diaphragm defect.

Acute intrathoracic gastric volvulus due to iatrogenic diaphragmatic hernia is a rare complication of thoracic and abdominal surgery. Therefore, it is difficult for a physician to diagnose it without prior personal experience. A delay in its diagnosis and treatment can have fatal consequences, such as gastric ischemia, perforation and hemorrhage. Considering these serious complications, we cannot overemphasize the importance of a detailed history, physical examination, and obtaining a delicate chest radiograph interpretation when the physician is faced with a patient presenting with vomiting and abdominal pain following abdominal or thoracic surgery.

\section{Disclosure Statement}

The authors of this case report have no conflicts of interest to disclose. 


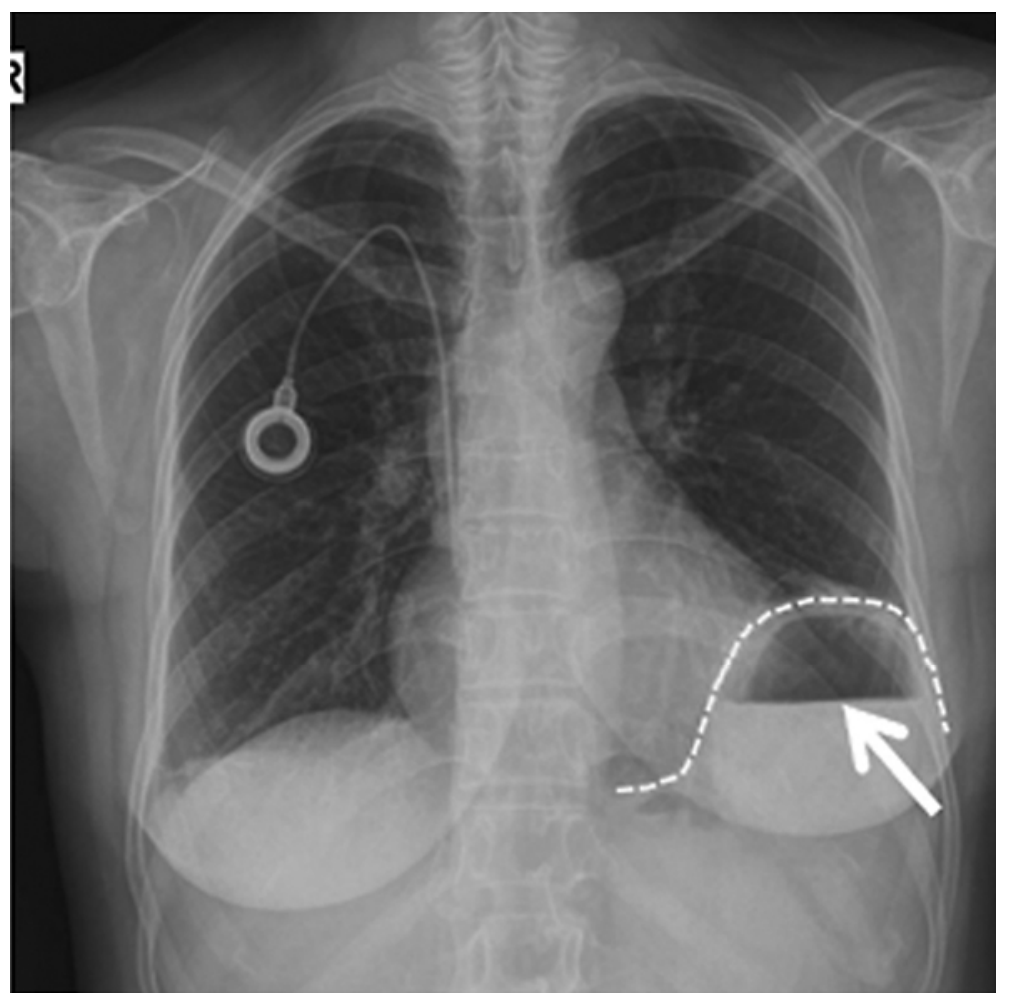

Fig. 1. Chest radiographic findings. Chest radiograph at admission demonstrates a high gastric air-fluid level on the left lower lung field (arrow) and elevated gastric contour (dotted line). The silhouette of heart and bony thorax shows no abnormal findings, and neither does the mediastinum. 

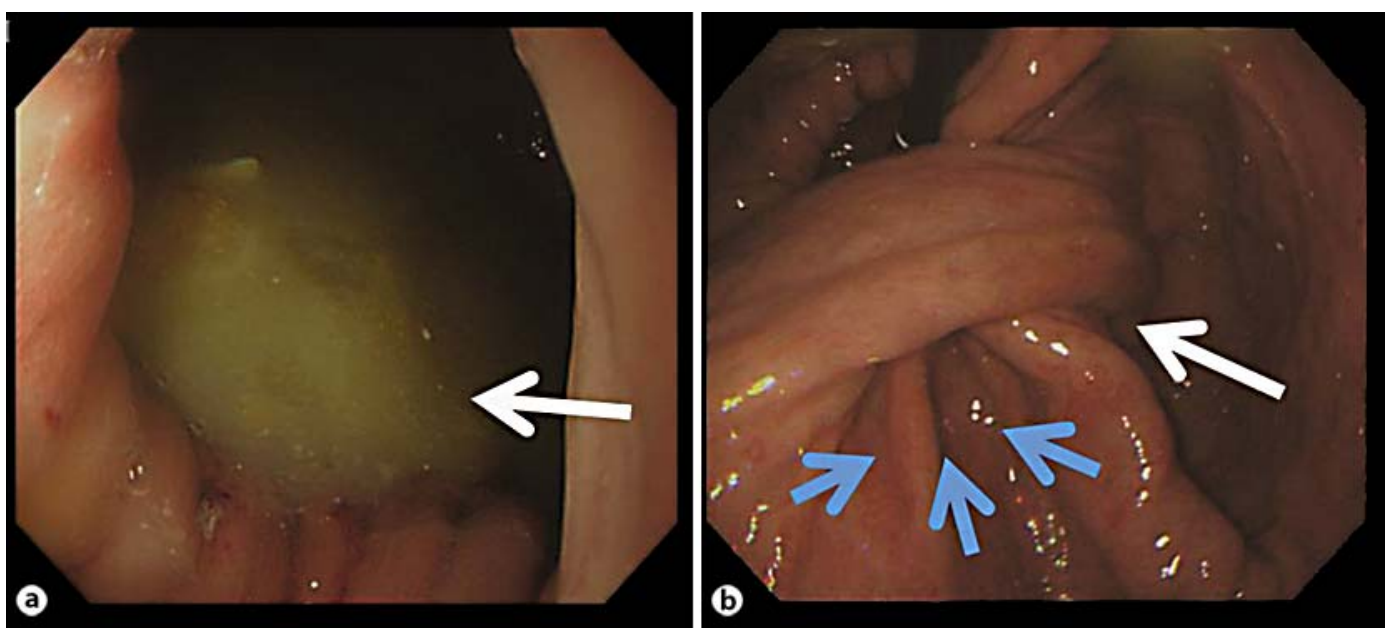

Fig. 2. Gastroscopic examination. a Gastroscopy demonstrates a large greenish fluid collection with a few food materials at the greater curvature side of the mid body of the stomach just after passing the esophagogastric junction (white arrow). There are no mucosal abnormalities such as hyperemia, fresh blood or old blood clots. b Retroflex view by gastroscopy revealed extreme twisting of the stomach (white arrow). The torsion of the stomach twisted the fundus and changed the anatomical structure so much that the fluid collection is not observed in the fundus, noticed in an ordinary situation (blue arrows).

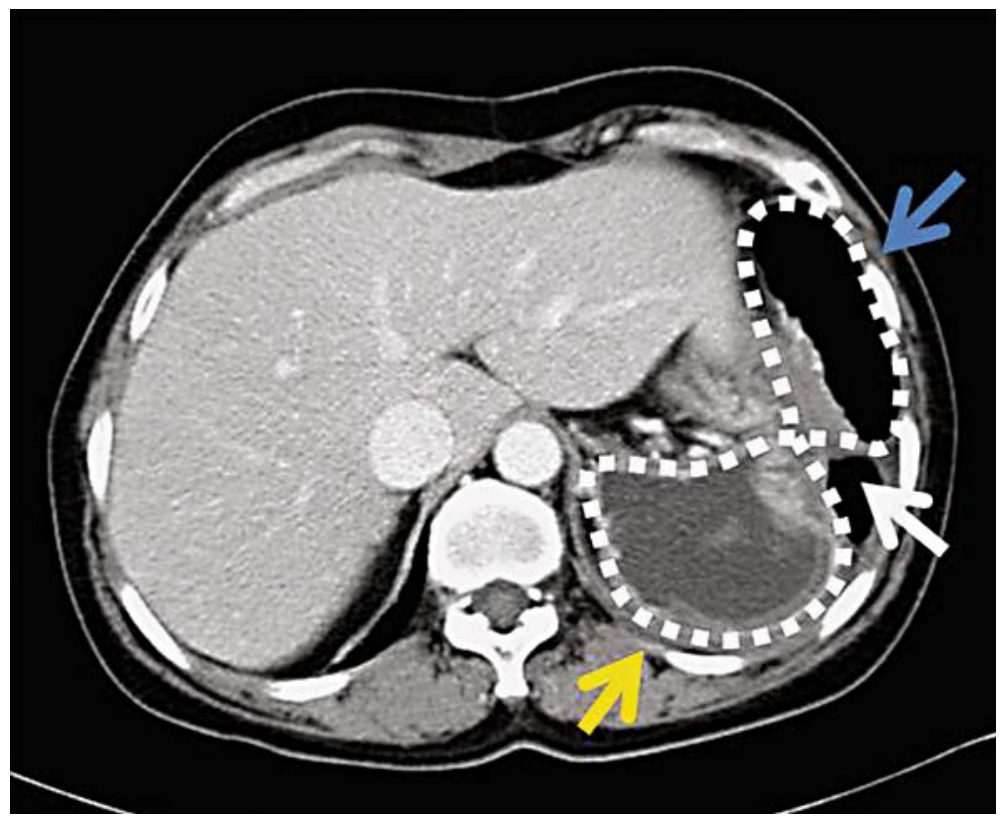

Fig. 3. CT findings. A chest CT scan demonstrates the butterfly-like gastric wall silhouette (dotted line) in the left lower hemithorax. One part of the stomach is filled with fluid (yellow arrow) and the other part with air (blue arrow). The twisted point is observed also (white arrow). 


\section{References}

$\checkmark 1$ Wasselle JA, Norman J: Acute gastric volvulus: pathogenesis, diagnosis, and treatment. Am J Gastroenterol 1993;88:1780-1784.

2 Iannelli A, Fabiani P, Karimdjee BS, Habre J, Lopez S, Gugenheim J: Laparoscopic repair of intrathoracic mesenterioaxial volvulus of the stomach in an adult: report of a case. Surg Today 2003;33:761-763.

-3 Axon PR, Whatling PJ, Dwerryhouse S, Forrester-Wood CP: Strangulated iatrogenic diaphragmatic hernia: a late diagnosed complication. Eur J Cardiothorac Surg 1995;9:664-666.

4 Alrabeeah A, Giacomantonio M, Gillis DA: Paraesophageal hernia after Nissen fundoplication: a real complication in pediatric patients. J Pediatr Surg 1988;23:766-768.

5 Testini M, Vacca A, Lissidini G, Di Venere B, Gurrado A, Loizzi M: Acute intrathoracic gastric volvulus from a diaphragmatic hernia after left splenopancreatectomy: report of a case. Surg Today 2006;36:981-984.

6 Maeng JH, Lee HS, Jang JG, Park BG, Nah BK, Kim YH, Jung SM, Cheon GJ: Acute gastric volvulus due to diaphragmatic hernia (in Korean). Korean J Gastroenterol 2003;42:544-548.

$\checkmark 7$ Milne LW, Hunter JJ, Anshus JS, Rosen P: Gastric volvulus: two cases and a review of the literature. J Emerg Med 1994;12:299-306.

8 Wu MH, Chang YC, Wu CH, Kang SC, Kuan JT: Acute gastric volvulus: a rare but real surgical emergency. Am J Emerg Med 2010;28:118.e5-118.e7.

9 Borchardt M: Zur Pathologie und Therapie des Magenvolvulus. Arch Klin Chir 1904;74:243-260.

10 Cherukupalli C, Khaneja S, Bankulla P, Schein M: CT diagnosis of acute gastric volvulus. Dig Surg 2003;20:497-499.

11 Gourgiotis S, Vougas V, Germanos S, Baratsis S: Acute gastric volvulus: diagnosis and management over 10 years. Dig Surg 2006;23:169-172.

12 Takanami I: Hernia of the diaphragm with gastric ulcer and volvulus: an unusual complication after diaphragmatic resection by VATS. Interact Cardiovasc Thorac Surg 2003;2:544-546.

13 Gean AD, DeLuca SA: Acute gastric volvulus. Am Fam Physician 1986;34:99-100.

14 Thorpe JA: Chronic gastric volvulus - aetiology and treatment. Br J Clin Pract 1981;35:161-162.

15 Cameron AE, Howard ER: Gastric volvulus in childhood. J Pediatr Surg 1987;22:944-947.

16 Smith RJ: Volvulus of the stomach. J Natl Med Assoc 1983;75:393-397. 